Five digit Project ID number: 60217

Project Title: Optically-Based Array Sensors for Selective In Situ Analysis of Tank Waste

Lead Principal Investigator: Gilbert M. Brown, Chemical and Analytical Sciences Division, Oak Ridge National Laboratory, P.O. Box 2008, MS 6119, Oak Ridge, TN 37831-6119

Email: browngm1@ornl.gov

Co-Principal Investigator(s): Reza Dabestani and Peter V. Bonnesen, Chemical and Analytical Sciences Division, Oak Ridge National Laboratory, P.O. Box 2008, MS 6119, Oak Ridge, TN 37831 and Professor David R. Walt, Department of Chemistry, Tufts University, Medford, MA

Post-Doctoral Fellows: Hai Feng Ji, ORNL and Caroline L. Schauer, Tufts University

Specific DOE Problems. There exist needs within the DOE for field instrumentation to perform real-time characterization and monitoring tasks. There is the need to provide fast sample concentration and preconditioning and for research in separations technologies for water/organic contaminant systems. In particular tank waste analysis provides great needs for systems that can reduce worker exposure and reduce turn around times. Idaho (ID-S104), Hanford (RL-WT09), and the Savannah River Site (SR00-2044) state needs for remote sensors for such elements as Cs137 and other radionuclides.

Research Objective. The objective of this research program is to conduct the fundamental research necessary to develop an array of chemically selective sensors, based on highly selective molecular recognition agents and highly sensitive fluorescence techniques, that can be coupled to fiber optics for remote analytical applications. These sensors will be of great value to DOE for the safe and cost-effective in situ characterization of high level waste tanks and other applications where remote sensing will prevent workers from being exposed to chemicals or radiation. The ability to detect and measure specific chemicals and radionuclides directly inside a high level waste tank using a remote sensing device could result in considerable benefits with regard to both cost savings and safety issues. In this approach to the design of sensors, agents for selective molecular recognition such as crown ethers are immobilized in an organic polymer matrix that mimics the organic medium in an aqueous nonaqueous extraction system.

Research Progress and Implications. Great progress has been made toward developing a fluorophore-selective ligand combination needed for $\mathrm{C} \mathrm{Cs}^{+}$selective fluorescence sensor in the two years and 5 months of this project. Calix[4]bis-crown-6-ethers in the 1,3-alternate conformation have been shown to possess a high degree of selectivity for cesium over sodium and moderate selectivity for cesium over potassium. Several systems involving the preparation of a new class of calixarene-based ionophores having attached fluorescent probe molecules were demonstrated. Cyanoanthracene attached to the benzyl group of a benzo crown was prepared as the first generation of a cesium selective optical sensor. This fluorophore has been shown to be an effective fluorescence turn-on group, exhibiting a ten-fold increase in fluorescence when $\mathrm{Cs}^{+}$binds to the oxygens of the benzo crown moeity. The fluorescence of the 9-cyanoanthracene is quenched by the benzo moiety owing to a Photoinduced Electron Transfer (PET) process. It has been shown that binding of the oxygen electron pairs of the benzo moiety upon metal ion complexation partially suppresses the PET process, allowing the fluorescence of the cyanoanthracene to increase. The synthesis of a 1,3-alternate di-deoxygenated calix[4]arene-(9cyano-10-anthrylmethyl)-benzocrown-6 (1) as the second generation cesium selective fluorescent probe has been accomplished by the route shown in the figure. This probe shows a 54 fold fluorescence enhancement response upon cesium complexation, which is the best result we have achieved to date. The selectivity ratios for this ligand to complex cesium over potassium and 
rubidium $\left(\mathrm{K}_{\mathrm{Cs}} / \mathrm{K}_{\mathrm{K}}\right.$ and $\left.\mathrm{K}_{\mathrm{Cs}} / \mathrm{K}_{\mathrm{Rb}}\right)$ are about ten fold higher than the first generation ligand. The observed selectivity ratios are consistent with data reported for other 1,3-alternate calix[4]dialkoxy-crown-6 derivatives. Work is in progress to translate these solution results to organic films. In a new concept for

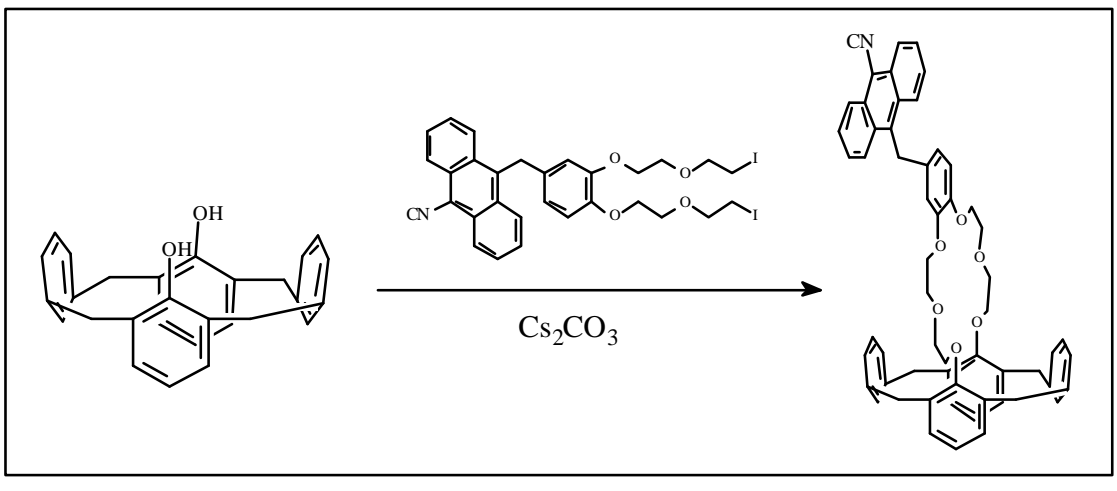
utilization of these sensor elements, the application to the development of logic devices has been pursued. The high selectivity exhibited by calix[4]crown-6 ethers towards the complexation of alkali metal ions has been exploited to synthesize a supramolecular fluorescent recognition agent capable of detecting both potassium and cesium ions in solution by PET. The new probe serves as a dual optical sensor to signal the presence of (a) potassium ions (as low as $10^{-6} \mathrm{M}$ ) in the presence of cesium ion (at concentrations as high as $5 \times 10^{-4} \mathrm{M}$ ) under basic conditions and (b) cesium ions $\left(10^{-7} \mathrm{M}\right)$ without any interference from potassium ion at concentrations as high as $10^{-5}$ $\mathrm{M}$ in acidic media. The newly synthesized probe also behaves as an integrated logic gate combining an OR and a NAND gate for signal transduction. This is the first example of an integrated molecular logic device with feed back to the input of one logic element from the output of another.

Planned Activities. During the remainder of this fiscal year, we will focus our attention on the evaluation of the polymeric organic matrix that will be used to attach (and/or imbed) the ionophores to the fiber optic or to polymer beads including the necessity of adding modifiers. The objective is to maximize both selectivity and loading as a function of matrix variation.

\section{Information Access.}

Hai-Feng Ji, Gilbert. M. Brown and Reza Dabestani "Calix[4]arene-based Cs+ selective optical sensor" Chem. Comm. 609, 1999.

Hai-Feng Ji, Reza Dabestani, Gilbert M. Brown and Robert L. Hettich "Spacer Length Effect on the Photoinduced Electron Transfer Fluorescent Probe for Alkali Metal lons" Photochem. Photobiol. 69, 513, 1999.

Hai-Feng Ji, Reza Dabestani, Robert L. Hettich, and Gilbert M. Brown “ Optical Sensing of Cesium Ions Using 1,3-Alternate Calix[4]-mono and di(Anthrylmethyl)Aza-Crown-6," Photochem. Photobiol. 70, 882-886, 1999.

Hai-Feng Ji, Reza Dabestani, Gilbert M. Brown and Richard A. Sachleben, "A new highly selective calix[4]crown-6 fluorescent cesium probe," Chem. Comm., in press.

Hai-Feng Ji, Reza Dabestani, and Gilbert M Brown, "A Supramolecular Fluorescent Probe Activated by Protons to Detect Cesium and Potassium ions. An Integrated Logic Gate Based on Photoinduced Electron Transfer (PET)," J. Am. Chem. Soc, submitted for publication. 